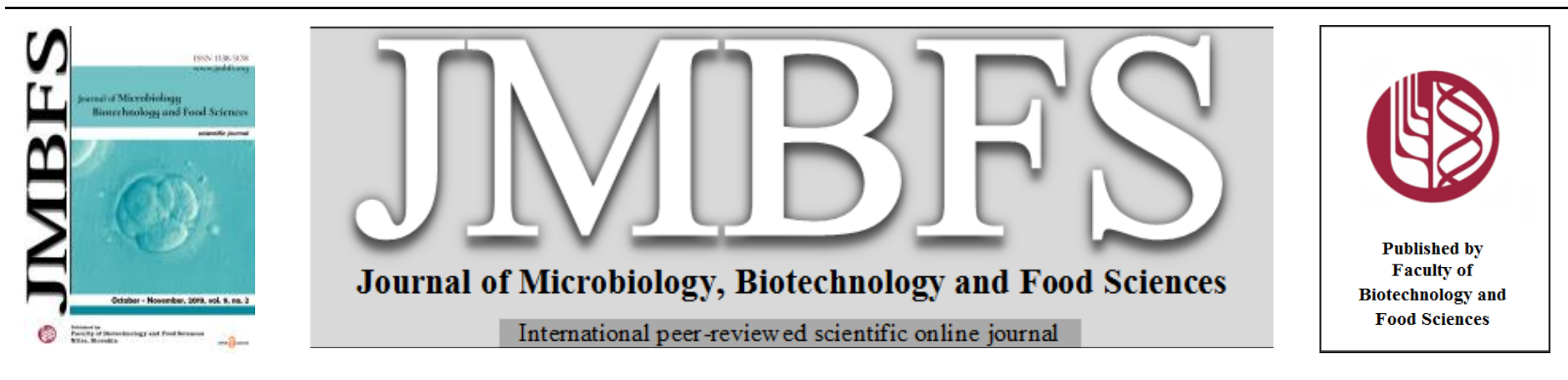

\title{
EFFICACY OF SEVERAL QTL AND mIRNA-SSR MARKERS TOWARDS HEALTH BENEFIT TRAITS IN AN ELITE DARJEELING TEA CULTIVAR
}

\author{
Anjan Hazra ${ }^{1,3}$, Shrutakirti Saha ${ }^{1}$, Rakesh Kumar ${ }^{2}$, Mrityunjay Chaubey $^{2}$, Chandan Sengupta ${ }^{3}$, Sauren Das ${ }^{1 *}$ \\ Address(es): Dr. Sauren Das, \\ ${ }^{1}$ Agricultural and Ecological Research Unit, Indian Statistical Institute, 203, B.T. Road, Kolkata 700108, India. \\ ${ }^{2}$ Darjeeling Tea Research and Development center, Kurseong, West Bengal 734203, India. \\ ${ }^{3}$ Department of Botany, University of Kalyani, Nadia, West Bengal 742 325, India. \\ *Corresponding author: sauren@isical.ac.in
}

doi: 10.15414/jmbfs.2020.9.4.700-704

ARTICLE INFO

Received 25. 4. 2019

Revised 29. 6. 2019

Accepted 27. 8. 2019

Published 3. 2. 2020

Regular article

open $\partial_{\text {ACCESS }}$

\begin{abstract}
Antioxidant and free radical scavenging ability of tea polyphenols are considered as health benefit traits that mainly contributed by tea catechins. Therefore understanding of genetic and molecular basis of this secondary metabolite production is of prime interest to facilitate the crop improvement program in tea plant efficiently. Some QTLs for these quality traits have already been available. In present study, 10 QTL linked SSRs and 14 newly developed microRNA based SSRs have been characterized in two Darjeeling tea cultivars having contrast characters and their progeny clone TS569 for validating possible association with catechin contents and antioxidant activities. Individual catechins and radical scavenging activity have been estimated by high performance liquid chromatography and UV-Vis spectrophotometry. Some of the polymorphic loci are indicated to be co-varied with such biochemical physiognomies. Characterization of microRNA based SSRs polymorphism are being the first time report in tea and discussed as ideal choice for further genotypic studies in tea.
\end{abstract}

Keywords: Antioxidant activity; catechins; microRNA; QTLs; SSRs; tea

\section{INTRODUCTION}

Tea is the highest consumed non-alcoholic beverage, made from young shoots of evergreen tea plant (Camellia sinensis). A significant share of revenue generation in major tea producing countries like China, India, Kenya, Sri Lanka by its production and export, thereby influencing the economic condition of the respective countries. In addition to its conventional refreshing ability, in recent years, the value added knowledge of tea health benefit properties have augmented the consumption of tea by health conscious people and increased the demand for quality tea marketing as well. The evidences point out the role of tea consumption in challenging the risk of neurological and cardiovascular disorder, diabetes, various types of cancer, oral disease even glaucoma too (Hayat et al., 2015; Hazra, Saha, et al., 2017; Khan \& Mukhtar, 2013; "Tea Advisory Panel, UK"). This increased awareness demand the attention of scientific community for breeding new cultivars with improved yield and quality according to the choice of end users. However, unlike most of other agricultural crops, heterozygous woody perennial nature of tea plant limit the conventional breeding and improvement program in tea (Hazra et al., 2018). The repetitive large genome ( 3GB) and polygenic inheritance nature of tea quality traits also hindered in generation of superior tea cultivar.

Generation and improvement of quality tea cultivars relies on screening the favorable strains with superior yield, quality and various abiotic and biotic stress tolerance ability (Kottawa-Arachchi et al., 2018). Recent trends of searching suitable biomarkers associated with desirable traits of agronomic crop is implemented in tea as well. However, the achievement in tea is still far from expectation than those of the other crops due to bottlenecks of life cycle in tea. Genome wide association studies and QTL mapping, in general, is the fundamental resource for understanding genomic background of complex phenotypic expression. Although a number of DNA markers have been reported on specific trait associated marker through mapping on different sets of breeding population (Bali et al., 2015; Kamunya et al., 2010; Koech et al., 2018; Ma et al., 2014; Tan et al., 2018), all of those might not be applicable for differentiation of worldwide genotypes as the genetic background may vary depending on origin and nature of segregating population (Tan et al., 2016). Therefore careful investigation and characterization of worldwide germplasm accessions and population is targeted to draw the roadmap towards achieving the goal of long term breeding program.
The primary value added quality underlying health benefit property of tea is mainly of its antioxidant properties and major bioactive components. Different polyphenols like catechin and flavonols which contribute up to $30 \%$ of the dry weight of the tender tea shoots (Pang et al. 2013). Among different catechin derivatives like epicatechin (EC), epicatechin gallate (ECG), epigallocatechin (EGC), epigallocatechin gallate (EGCG), EGCG is the most abundant, up to 70\% of the total tea catechins (Saravanan et al., 2005) and these possess significant antioxidant and radical-scavenging activity (Tounekti et al., 2013; Zaveri, 2006). The major polyphenols like EC, EGC, ECG and EGCG are reported to have anti-arthritic effect as well. Hence, increasing the catechin content has become a major challenge for tea breeding program (Ma $\boldsymbol{e t}$ al., 2014). Being highly abundant, polymorphic, locus specific, co-dominantly inherited and reproducible the simple sequence repeat or SSR or microsatellite markers are most popular and versatile type in plant genetic mapping studies Among the two types of microsatellite markers (genic and genomic) genic SSRs or expressed sequence derived markers are the utmost compelling for tagging and mapping of genes and QTLs (Varshney et al., 2005). Considering the fact, present study aim to explore and characterize some DNA marker and corresponding biochemical phenotype in order to facilitate marker trait association study in tea. One elite Darjeeling tea cultivar TS569 (released through bi-parental cross between Ambari Vegetative-2 and Tukdah-78) was considered for evaluation of the SSR markers and antioxidant trait using spectrophotometry and high performance liquid chromatography.

\section{MATERIALS AND METHODS}

\section{Plant material and habitat}

Two popular Darjeeling tea cultivars AV-2 and T-78 and a set of individual $(\mathrm{n}=10)$ from their pseudo-testcross progeny TS-569 of two year maturity (Figure $1 \mathrm{~A}-\mathrm{C})$ were selected for the current study. Both the parents and their progeny (indicated as TS01 - TS10 hereafter) are being maintained at experimental field of Darjeeling Tea Research and Development Center, Kurseong. The study site is located between $26.8821^{\circ} \mathrm{N}, 88.2789^{\circ} \mathrm{E}$ with an elevation of 1530 meter above sea level and the annual average rainfall is $3235 \mathrm{~mm}$. Temperature of the region varies from $8-20^{\circ} \mathrm{C}$. 


\section{Extraction of secondary metabolites}

The tender 'two and a buds' of selected individuals were collected from the field immediately preserved at ice chilled condition for subsequent laboratory experimental works. Tea specific secondary metabolites extraction was performed following a modified protocol after Liu et al. (2015). Fresh lea tissues $(500 \mathrm{mg})$ were homogenized in a mortar and pestle and extracted in $5 \mathrm{ml}$ of $70 \%$ methanol $(\mathrm{v} / \mathrm{v})$ with $1 \%$ formic acid $(\mathrm{v} / \mathrm{v})$. The tubes containing homogenized tissue were heated in a water bath at $80^{\circ} \mathrm{C}$ for $15 \mathrm{~min}$ followed by centrifugation at $4{ }^{\circ} \mathrm{C}$ and $10,000 \mathrm{rpm}$ for $15 \mathrm{~min}$ in a refrigerator centrifuge (Eppendoff 5418R). The clear supernatants were passed through a $0.45 \mu \mathrm{m}$ membrane filter and stored at $-20{ }^{\circ} \mathrm{C}$ avoiding light exposure until further analyses. The standard reference materials of catechin, epicatechin, epicatechin gallate, catechin gallate, epigallo catechin, epigallocatechin gallate and caffeine were purchased from Sigma-Aldrich, dissolved in $70 \%$ methanol as $2000 \mu \mathrm{g} / \mathrm{m}$ stock concentration and stored at the previously mentioned condition. Standard reference materials were diluted from the stock when needed as working solution.

\section{Quantification of catechin derivatives and caffeine by HPLC}

High performance liquid chromatography has been carried out in a Waters Alliance MDLC Separations module and dual absorbance detector (Waters e2695-2487) equipped with a C18 reverse phase silica based column (X-Tera) with $4.6 \times 250 \mathrm{~mm}$ dimension at $35^{\circ} \mathrm{C}$. The double gradient elution system was standardized with mobile phase consisting of solvent A ( $10 \%$ acetonitrile including $0.1 \%$ trifluoroacetic acid in HPLC grade $\left.\mathrm{H}_{2} \mathrm{O}\right)$ and solvent B (90\% acetonitrile with $0.1 \%$ trifluoroacetic acid). The gradient elution profile for mobile phases are as follows: 0 to 8 minute $100 \% \mathrm{~A}$ and $0 \% \mathrm{~B}, 8$ to 14 minute $70 \% \mathrm{~A}$ and $30 \% \mathrm{~B}, 15$ to 25 minute $100 \% \mathrm{~A}$ and $0 \% \mathrm{~B}$ at $1 \mathrm{ml} / \mathrm{min}$ flow rate. The detection wavelength have been set at $280 \mathrm{~nm}$ and $254 \mathrm{~nm}$ respectively. Different Catechin, catechin derivatives and caffeine peaks have identified (Figure 1D) and quantified based on comparing retention times and peak area of the same obtained from standard reference materials respectively. The downstream analyses have been performed using Empower Pro software from Waters ltd.

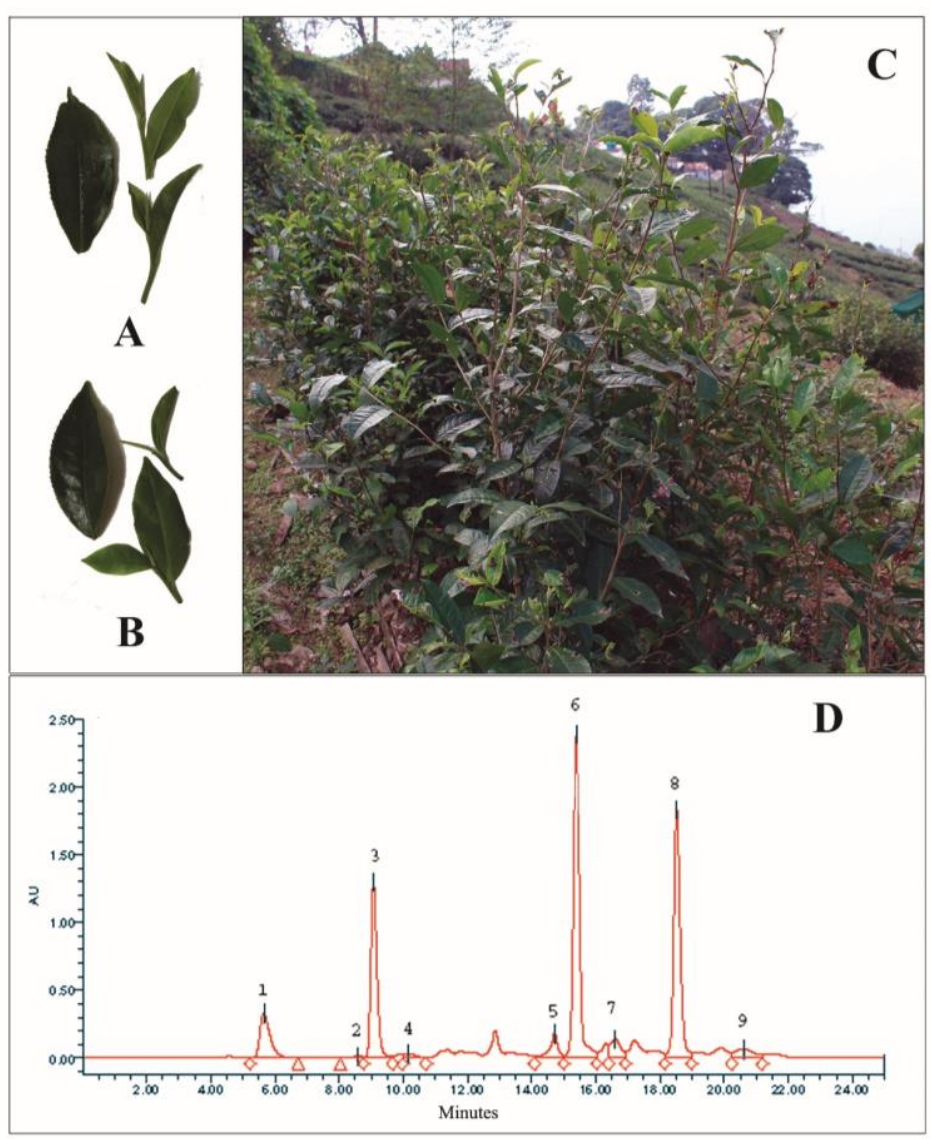

Figure 1 (A-D): Plant samples used in the study, Mature leaf and two and a bud of A - AV2, B - T78, C - Individuals of mapping population of TS569, D Representative HPLC chromatogram with individual catechin peaks - 1 (gallocatechin; GC), 2 (epigallocatechin; EGC), 3 (caffeine), 4 (catechin; C), 5 (epicatechin; EC), 6 (epigallocatedchin gallate; EGCG), 7 (gallocatechin gallate; GCG), 8 (epicatechin gallate; ECG) and 9 (catechin gallate, CG)
Determination of free radical (ROS/RNS) scavenging activity

Superoxide radical, a reactive oxygen species scavenging activity has been measured by the nitro blue tetrazolium (NBT) reduction method (Fontana et al., 2001). The non-enzymatic phenazinemethosulfate (PMS) and nicotinamide adenine dinucleotide $(\mathrm{NADH})$ system generates superoxide radicals, which in turn reduce (NBT) to a purple formazan. The reaction mixture consisted with 20 $\mathrm{mM}$ phosphate buffer ( $\mathrm{pH} 7.4), 73 \mu \mathrm{M}$ NADH, $50 \mu \mathrm{M}$ NBT, $15 \mu \mathrm{M}$ PMS and test sample solution. After incubation for $5 \mathrm{~min}$ at ambient temperature, the absorbance of generated formazanhas been measured at $562 \mathrm{~nm}$ of wavelength. Radical scavenging activity of the samples have been expressed by the calculated IC50 value through standard calculation method.

The nitric oxide radical, a reactive nitrogen species scavenging ability has been quantified by the Griess Illosvoy reaction (Garratt, 2012). Different concentration of samples have been allowed to react with $10 \mathrm{mM}$ Sodium nitropruside in phosphate buffered saline ( $\mathrm{pH}$ 7.4). Following incubation at $25^{\circ} \mathrm{C}$ for $150 \mathrm{~min}, 0.5 \mathrm{ml}$ of the incubated solution has been mixed with $1 \mathrm{ml}$ sulfanilamide $(0.33 \%$ in $20 \%$ glacial acetic acid) and allowed to stand for 5 minute. Thereafter $1 \mathrm{ml}$ of napthylethylenediamine dihydrochloride (NED) $(0.1 \% \mathrm{w} / \mathrm{v})$ was added to it and incubated the mixture at $25^{\circ} \mathrm{C}$ for $30 \mathrm{~min}$. The pink chromophore generated in the resultant mixture has been measured spectrophotometrically at $540 \mathrm{~nm}$ of wavelength. Radical scavenging activity of the samples have been expressed by the calculated IC50 value through standard calculation method.

\section{Isolation of genomic DNA}

Total genomic DNA from all plant samples have been isolated using a DNeasy Plant kit (Qiagen). A total amount of 100-200 mg Fresh leaf tissues have been homogenized into fine powder with liquid nitrogen and transferred to tube containing $400 \mu \mathrm{l}$ buffer AP1 (included in kit), $40 \mu 1 \beta$-mercaptoethanol and $8 \mu \mathrm{l}$ RNAse $\mathrm{A}$ and placed in a water bath at $65^{\circ} \mathrm{C}$ for 10 minutes. The remaining steps have been followed as the manufacturer's protocol. Finally the DNA samples have been eluted in $100 \mu$ l elution buffer and stored at $-20{ }^{\circ} \mathrm{C}$ until further use. Quality and quantity of the isolated DNA are being checked through agarose gel electrophoresis and a Qubit 2.0 (Invitrogen) fluorometer assay respectively.

\section{Characterization of QTL and miRNA based SSR markers}

A set of 10 different catechin QTL linked SSR markers (Ma et al., 2014) and 14 microRNA precursor based SSR flanking primers, previously designed in this laboratory (Hazra, Dasgupta, et al., 2017) were taken into consideration for genotyping the selected individuals of F1 progeny and their parents AV2 and T78 cultivars (Table 1). The PCR mixture contained of $25 \mu \mathrm{L}$ total volume: $5 \mu 15 \times$ flexi buffer, $0.5 \mathrm{mM} \mathrm{MgCl} 2,4 \mathrm{mM}$ dNTPs, $10 \mu \mathrm{M}$ of each primer, $1 \mathrm{U}$ GoTaq DNA polymerase and $10 \mathrm{ng}$ genomic DNA (Promega, USA). Amplification of QTL-SSRs has been performed according to the method of (Zhao et al., 2008) using the following reaction conditions: $4 \mathrm{~min}$ at $94^{\circ} \mathrm{C}$ for initial denaturation, followed by 35 cycles of $94^{\circ} \mathrm{C}$ for $30 \mathrm{~s}$, annealing temperature for $30 \mathrm{~s}, 72^{\circ} \mathrm{C}$ for $30 \mathrm{~s}$, with a final extension at $72^{\circ} \mathrm{C}$ for $7 \mathrm{~min}$. Amplification profile for miRNA based SSRs are being followed as: initial denaturation at $95{ }^{\circ} \mathrm{C}$ for $5 \mathrm{~min}$ followed by 34 cycles of $95{ }^{\circ} \mathrm{C}$ for $1 \mathrm{~min}, 55^{\circ} \mathrm{C}$ for $1 \mathrm{~min}$ and $72{ }^{\circ} \mathrm{C}$ for $2 \mathrm{~min}$ (Ganie \& Mondal, 2015). The final extension was at $72{ }^{\circ} \mathrm{C}$ for $10 \mathrm{~min}$. The amplicons have been separated in a 3\% Agarose gel with ethidium bromide staining, visualized and photographed in a gel documentation system (Biostep, Germeny) equipped with a Olympus C7070 camera. Polymorphic bands for each primers have been manually scored and utilized the scored data for correlation analyses and interpretations.

\section{RESULT}

\section{Catechins and caffeine contents}

A wide range of catechins, EC, CG, EGC, ECG, EGCG and caffeine content were observed (Figure 2) among the sampled individuals. The most abundant of them is EGCG and the least present is CG. Out of the two parents, T78 has more amount of catechins than that of AV2. Wide variation in catechins content were observed among the individuals of F1 progeny. The F1 progeny also exhibits similar characteristics, like EGCG is the most abundant and CG to be the least. TS05 from F1 progeny has an overall higher amount of catechins. The highest amount of EGCG and caffeine is present in TS02. The catechin and caffeine content is mostly higher in F1 progeny than the parents, whereas CG, ECG, EGC, EGCG are mostly in lower amount as to the parents. Pearson's correlations between catechin compounds were calculated and appended in Table 2 It is revealed from the result that the individual catechins correlated significantly with each other, having strong positive correlation between EC and ECG $(r=0.758$, $\mathrm{P} \leq 0.01), \mathrm{CG}$ and ECG $(\mathrm{r}=0.825, \mathrm{P} \leq 0.01), \mathrm{CG}$ and EGCG $(\mathrm{r}=0.824, \mathrm{P} \leq 0.01)$, ECG and EGCG $(r=0.869, \mathrm{P} \leq 0.01)$. 
Table 1 Primers used in the study

\begin{tabular}{|c|c|c|c|c|c|c|}
\hline Type & $\begin{array}{l}\text { Marker } \\
\text { ID }\end{array}$ & $\begin{array}{l}\text { Repeat } \\
\text { motif }\end{array}$ & Forward primer sequence & Reverse primer sequence & $\begin{array}{l}\text { Ta } \\
\left({ }^{\circ} \mathrm{C}\right)\end{array}$ & $\begin{array}{l}\text { Expected allele } \\
\text { size (bp) }\end{array}$ \\
\hline \multirow{10}{*}{$\begin{array}{l}\text { QTL- } \\
\text { SSR }\end{array}$} & TM376 & (AGATGG)3 & GACGACTTGGAGGGTACGAG & CACGTATCCTTTGGCCTGTT & 52 & 231 \\
\hline & TM546 & (ATCTCA) 3 & TTGAAGTTAGCCAAGGCATC & TCCCAATCCCTTTAGTGTGC & 52 & 115 \\
\hline & TM623 & (CTT)6 & TCCCAATCATCATCATCGAA & GGTTCCCATTGGTTTGTGTC & 52 & 141 \\
\hline & TM586 & (TGAGAT)4 & TCGATAATACCCCCAACCAA & TCTCTCTCAAAAGTGGGCGT & 52 & 186 \\
\hline & TM560 & (TC)10 & CAAGTTTGCGAGTCCTCCTC & GCCCACGAGGTTAGATGTGT & 58 & 185 \\
\hline & TM453 & (TTC)6 & AAGTCACAACACCACCACCA & GAGGCAGCGATAGTACCAGG & 52 & 268 \\
\hline & TM340 & $(\mathrm{ACC}) 9$ & TGAGGACGACGAAGGAT & TCCGAGCCACCGAACAT & 52 & 113 \\
\hline & TM399 & (TC) 8 & TCTCTGCTCTGCTGCTTTCA & CGATCTGGTTGGGACAAAGT & 54 & 107 \\
\hline & TM412 & (AG)14 & GCAGGCTTCAAGGTTTTCAG & TGTTGTTCTGGCGTTGAGAG & 52 & 215 \\
\hline & TM435 & (CTC)6 & GGTGGTGTGGTTTAGAGGGA & TTCATCTTGGGGAGCAATTC & 52 & 242 \\
\hline \multirow{14}{*}{$\begin{array}{l}\text { miRNA- } \\
\text { SSR }\end{array}$} & miSSR01 & (CGC)3 & GGAGTGAAGGAGGGAAGA & CCGAGAGAATAATAAGGAGGA & 53 & 205 \\
\hline & miSSR02 & $(\mathrm{GAG}) 3$ & AGTGATTATTGGTGGTGGTC & TCTCAACCAATTCAACAAGTC & 52 & 152 \\
\hline & miSSR03 & (TA)2 & CTGTTACTGCAGCTTAACCAA & AAATATGCTGCTCATTCAAAC & 52 & 152 \\
\hline & miSSR04 & $(\mathrm{GGC}) 3$ & AGAAGATGTCTCAGGGAAGAG & ACACAAGTATGTCACCAGCTC & 54 & 177 \\
\hline & miSSR05 & (GTGGA)5 & GAACCTTTCCTCCAGAATTTA & TCACATTTAGCTTTTCACTCC & 50 & 162 \\
\hline & miSSR06 & (GATGAC)6 & ACGATGGTGGTTATGAATATG & GGGTTTTTGTTAAGTTGTTCA & 50 & 139 \\
\hline & miSSR07 & (TTC)3 & AGACAAAAACCAAGGCTAGAT & CATCTTGTGCAGATCTCAGTT & 52 & 149 \\
\hline & miSSR08 & (CTC)3 & TGATGATGAGGAAGGAGATAA & TTGCTTTAGTGAAACAACTCC & 51 & 136 \\
\hline & miSSR09 & $(\mathrm{GA}) 2$ & TAGAACTTGCAGAGAGAATCG & TACACATTGGGAAGAAGAAGA & 51 & 152 \\
\hline & miSSR10 & (TTC) 3 & AGATACACATTGGGAAGAAGG & TAGAACTTGCAGAGAGAAACG & 52 & 149 \\
\hline & miSSR11 & $(\mathrm{GGA}) 3$ & AGACACAGGCAGACATAGAGA & AAGATGCGATGAGATCAGATA & 55 & 165 \\
\hline & miSSR12 & $(\mathrm{AAC}) 3$ & CGTTAGGCTATTTTGTTTCAA & TTCTGTCAATCATCCAATTTC & 49 & 159 \\
\hline & miSSR13 & (TC) 2 & TACTTCCAACCAAACACAAGT & TAGCTTACCACCTCAATCAAA & 52 & 168 \\
\hline & miSSR14 & (TCTA) 4 & CTCCTGTACACTCTCTCTCTCC & GATGAACAGCATAGGTATCCA & 55 & 127 \\
\hline
\end{tabular}

\begin{tabular}{|c|c|c|c|c|c|c|}
\hline C & EC & CG & ECG & EGC & EGCG & Caffeine \\
\hline 12.8 & 39.2 & 0.1 & 13.9 & 4 & 234.8 & 5.9 \\
\hline 13.8 & 92.7 & 0.5 & 39.1 & 9.8 & 499.9 & 9.7 \\
\hline 6.9 & 15.9 & 0 & 6.7 & 4.2 & 168.9 & 5.6 \\
\hline 26.3 & 21.8 & 0.4 & 12.7 & 7.3 & 361.7 & 18.1 \\
\hline 4.9 & 9.5 & 0 & 8.7 & 2.9 & 168.4 & 5.9 \\
\hline 5.7 & 12.1 & 0.1 & 4.8 & 5.9 & 110.9 & 5.2 \\
\hline 24.4 & 51.5 & 0.3 & 17.7 & 27.4 & 305.9 & 15.1 \\
\hline 2.4 & 5.7 & 0 & 1.6 & 3.8 & 143.8 & 4.7 \\
\hline 0.2 & 58 & 0 & 2.7 & 6.2 & 220.3 & 16.8 \\
\hline 29 & 22.9 & 0 & 2.5 & 6 & 79.3 & 5.7 \\
\hline 15.7 & 19.1 & 0.1 & 13.2 & 8.3 & 318.2 & 10.1 \\
\hline 25.4 & 50.2 & 0.1 & 12.9 & 11.5 & 170.3 & 5.4 \\
\hline
\end{tabular}

Figure 2 Concentrations of catechins and caffeine in $\mu \mathrm{g} / \mathrm{ml}$ among studied samples

\section{ROS/RNS scavenging activity}

Free radical scavenging activity has been determined of the parents and the F1 progeny and obtained data presented by calculated IC50 values (inhibitory concentration to scavenge 50\% of radicals). More IC50 value indicating to less scavenging potential or anti-oxidant capacity present in the sample. It is observed that AV2 and T78 has similar scavenging activity except T78 has slightly higher nitric oxide scavenging activity, in turn, the antioxidant property attribute to be low (Table 3 ). The progeny shows simila characteristics for superoxide scavenging efficiency as the parents whereas, nitric oxide scavenging activity is similar to that of AV2.

Table 2 Correlation among different catechin concentrations

\begin{tabular}{llllll}
\hline & C & EC & CG & ECG & EGC \\
\hline EC & 0.184 & & & & \\
\hline CG & 0.402 & $.587^{*}$ & & & \\
\hline ECG & 0.267 & $.758^{* *}$ & $.825^{* *}$ & & \\
\hline EGC & 0.503 & 0.439 & 0.498 & 0.386 & \\
\hline EGCG & 0.197 & $.657^{*}$ & $.824^{* *}$ & $.869^{* *}$ & 0.357 \\
\hline
\end{tabular}

*. Correlation is significant at the 0.05 level (2-tailed).

**. Correlation is significant at the 0.01 level (2-tailed).

Table 3 Antioxidant activity of studied samples

Sample

Free radical scavenging activity $\left(\mathrm{IC}_{50}\right)$ Nitric oxide

\begin{tabular}{lcc}
\hline AV2 & $0.532 \pm 0.008$ & $0.322 \pm 0.003$ \\
\hline T78 & $0.703 \pm 0.007$ & $0.321 \pm 0.003$ \\
\hline TS01 & $0.498 \pm 0.01$ & $0.322 \pm 0.003$ \\
\hline TS02 & $0.585 \pm 0.029$ & $0.324 \pm 0.003$ \\
\hline TS03 & $0.524 \pm 0.008$ & $0.345 \pm 0.006$ \\
\hline TS04 & $0.541 \pm 0.024$ & $0.32 \pm 0.006$ \\
\hline TS05 & $0.516 \pm 0$ & $0.323 \pm 0.004$ \\
\hline TS06 & $0.589 \pm 0.024$ & $0.334 \pm 0.007$ \\
\hline TS07 & $0.588 \pm 0.005$ & $0.337 \pm 0.002$ \\
\hline TS08 & $0.583 \pm 0.009$ & $0.317 \pm 0$ \\
\hline TS09 & $0.535 \pm 0.004$ & $0.319 \pm 0.001$ \\
\hline TS10 & $0.561 \pm 0.013$ & $0.33 \pm 0.001$ \\
\hline
\end{tabular}

Molecular marker based polymorphism

All the QTL-SSR markers tested in present work, produce clear and reproducible band in both the parents and their progeny genotyped at their respective loci. A total 20 alleles have been scored with 10 SSR markers among which 14 are polymorphic $(70 \%)$ in nature. Maximum number of alleles (4) produced by TM560 followed by TM340 and TM399 with 3 loci each (Figure 3). In case of 14 miRNA based SSR markers, 22 loci have been scored, among which 15 are polymorphic $(68 \%)$. Maximum number of loci (3 loci) reproduced by miSSR04 and most others with two of each.
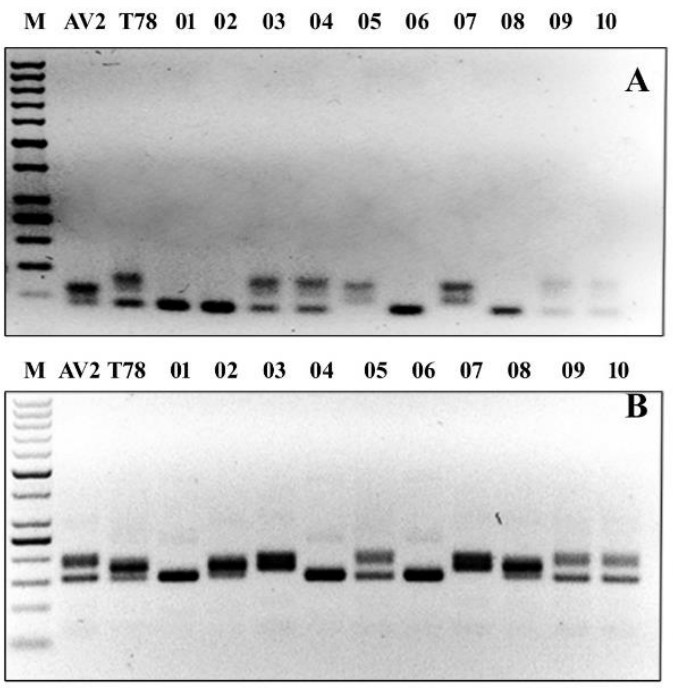

Figure 3 Representative agarose gel picture of A- QTL-SSR marker TM399 and B - miRNA-SSR-03 


\section{Marker trait correlation analysis}

Point biserial correlation coefficients have been observed between different markers and biochemical traits of the tea plant (shown in Table 4). The correlation between TM399 and nitric oxide $(\mathrm{r}=0.701, \mathrm{P} \leq 0.05)$, TM399 and caffeine $(\mathrm{r}=-0.655, \quad \mathrm{P} \leq 0.05), \quad \mathrm{TM} 399$ and $\mathrm{EGC}(\mathrm{r}=-0.616$, $\mathrm{P} \leq 0.05)$, TM435 and nitric oxide $(\mathrm{r}=0.685, \mathrm{P} \leq 0.05)$, TM560 and superoxide $(\mathrm{r}=$

Table 4 Significantly correlated bands with biochemical parameters

\begin{tabular}{|c|c|c|c|c|c|c|c|c|c|c|c|}
\hline 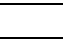 & Marker & Nitric oxide & Superoxide & Caffeine & $\mathbf{C}$ & EC & EGCG & ECG & CG & EGC & TC \\
\hline C06 & TM399 & $.701^{*}$ & & $-.655^{*}$ & & & & & & $-.616^{*}$ & \\
\hline C11 & TM435 & $.685^{*}$ & & & & & & & & & \\
\hline $\mathrm{C14}$ & TM560 & & $-.695 *$ & & & & & & & & \\
\hline D01 & $\mathrm{mi01}$ & & & & & $-.726^{* *}$ & $-.702 *$ & $-.856 * *$ & $-.669 *$ & & $-.733 * *$ \\
\hline D08 & $\operatorname{mi05}$ & & & & & $.600^{*}$ & & & & & \\
\hline D22 & $\operatorname{mi14}$ & & & & $.723 * *$ & & & & & & \\
\hline
\end{tabular}

**. Correlation is significant at the 0.01 level (2-tailed).

*. Correlation is significant at the 0.05 level (2-tailed).

\section{DISCUSSION}

Identification and QTL for agronomically desired traits and subsequent validation of marker-trait associations are key steps in developing of improved tea cultivars. As a woody perennial crop, tea agronomic traits are being attempted to link with molecular markers in order to select individuals with desired physiognomies at early stages of growth (Koech et al., 2018; Ma et al., 2014; Tan et al., 2016) Different secondary metabolites like catechin, caffeine concentration and other antioxidant traits are considered as the major contributor of tea quality in terms of its health benefit aspect. Ma et al. (2014) first identified 25 QTLs for catechins content in tea plant and reported 12 SSR markers closely linked to these QTLs. In present study 10 markers among the earlier study (Ma et al., 2014) are cross validated in biparental mapping population of TS569 (AV2×T78). Among the tested markers, almost $70 \%$ of the resulting loci are polymorphic. However, only one of the validated markers TM399 has been found to significantly associate with EGC content among studied individuals. Though, the above marker and the other two markers (TM435 and TM560) are significantly associated with studied traits like caffeine content and radical scavenging activity. Reduced efficiency of other earlier reported markers can be attributed to the reasons, i) QTLs are critically subjective to different growing environments and several abiotic conditions during sampling time may have responsive role in altered phenotype (Chen et al., 2010; Collard et al., 2005), ii) small sample size of studied mapping population minimal statistical support for detection of QTLs. To overcome this bottlenecks long term phenotyping with a considerably large population has to be taken into account in subsequent research. In recent times, SSR motif variation within microRNA precursor has become a marker of choice for trait association study. Some earlier studies suggested that length variations of the repeats in miRNA precursor gene interfere the stem-loop structure formation of premature miRNA and their synthesis which may lead to develop trait specific miRNA based SSR markers in plants (Ganie \& Mondal, 2015; Mondal \& Ganie, 2014). Mondal and Ganie (2014) reported that SSRs within rice miRNA genes could distinguish salt tolerant and susceptible genotypes and also figured out the genetic diversity of salt sensitive genotypes. In tea, trait association study with miRNA precursor based SSR markers still unexplored. Very recently, 18 SSR motifs are found to be present in 13 predicted miRNAs from tea expressed tag sequence database and 14 primer pairs were designed from the SSR flanking region (Hazra, Dasgupta, et al., 2017). Current study experimentally validated all 14 miRNA based SSRs and three of them (miSSR-01, miSSR-05 and miSSR14) has been found to be associated with certain catechin contents. Most significantly miSSR-01 has the excellent co-variability and reverse correlation with almost all catechin derivatives. Further investigation on miSSR-01points to a miR164 family precursor gene. This miR164 family members are conserved microRNAs and expressed in the leaves of tea (Prabu \& Mandal, 2010) Jeyaraj et al. (2017) reported that expression of Camellia sinensis miR164a downregulates the NAC transcription factor gene which controls various biotic and abiotic stress response. Another member of the family miR164b is involved in phenylpropanoid biosynthesis. Another report suggested that miR164b has potential role in regulating a catechin pathway gene anthocyanidin synthase $(A N S)$ by inhibiting its translation (Ping et al., 2017). Therefore the repeat motif variation in the said microRNA precursor might have vital role in its formation and function which in turn influence the downstream biochemical expression and ultimately tea leaf catechin contents. As a whole, this study suggests along with reported QTL-SSR markers newly developed miRNA based SSRs can be suitable choice for trait association mapping study in tea. Moreover, highly polymorphic reproducible and regulatory non coding RNA specific SSRs are ideal for specific and sensitive genotypic application.

Acknowledgement: The author are thankful to the Project Director, DTR \& DC for providing necessary permission regarding field work.
$-0.695, \mathrm{P} \leq 0.05)$. The correlations between the microRNA SSR markers and traits have also been observed. The correlations between mi01 and EC, EGCG, ECG CG, TC are $(r=-0.726, P \leq 0.01),(r=-0.702, P \leq 0.05),(r=-0.856, P \leq 0.01),(r=-$ $0.669, \leq 0.05),(\mathrm{r}=-0.733, \mathrm{P} \leq 0.01)$, respectively. The correlation between mi05 and $\mathrm{EC}(\mathrm{r}=0.600, \mathrm{P} \leq 0.05)$, mi14 and $\mathrm{C}(\mathrm{r}=0.723, \mathrm{P} \leq 0.01)$.

\section{REFERENCES}

Bali, S., Mamgain, A., Raina, S. N., Yadava, S. K., Bhat, V., Das, S., . . Goel, S (2015). Construction of a genetic linkage map and mapping of drought tolerance trait in Indian beveragial tea. Molecular breeding, 35(5), 112 https://doi.org/10.1007/s11032-015-0306-5

Chen, X., Zhao, F., \& Xu, S. (2010). Mapping environment specific quantitative trait loci. Genetics. https://doi.org/10.1534/genetics.110.120311

Collard, B., Jahufer, M., Brouwer, J., \& Pang, E. (2005). An introduction to markers, quantitative trait loci (QTL) mapping and marker-assisted selection for crop improvement: the basic concepts. Euphytica, 142(1-2), 169-196. https://doi.org/10.1007/s10681-005-1681-5

Fontana, M., Mosca, L., \& Rosei, M. A. (2001). Interaction of enkephalins with oxyradicals1. Biochemical Pharmacology, 61(10), 1253-1257. https://doi.org/10.1016/S0006-2952(01)00565-2

Ganie, S. A., \& Mondal, T. K. (2015). Genome-wide development of novel miRNA-based microsatellite markers of rice (Oryza sativa) for genotyping applications. Molecular breeding, 35(1), 51. https://doi.org/10.1007/s11032-015$\underline{0207-7}$

Garratt, D. C. (2012). The quantitative analysis of drugs: Springer Science \& Business Media.

Hayat, K., Iqbal, H., Malik, U., Bilal, U., \& Mushtaq, S. (2015). Tea and its consumption: benefits and risks. Critical reviews in food science and nutrition, 55(7), 939-954. https://doi.org/10.1080/10408398.2012.678949

Hazra, A., Dasgupta, N., Sengupta, C., \& Das, S. (2017). Extrapolative microRNA precursor based SSR mining from tea EST database in respect to agronomic traits. BMC research notes, 10(1), 261 https://doi.org/10.1186/s13104-017-2577-x

Hazra, A., Dasgupta, N., Sengupta, C., \& Das, S. (2018). Next generation crop improvement program: Progress and prospect in tea (Camellia sinensis (L.) O Kuntze). Annals of Agrarian Science. https://doi.org/10.1016/j.aasci.2018.02.002 Hazra, A., Saha, J., Dasgupta, N., Sengupta, C., Kumar, P. M., \& Das, S. (2017). Health-benefit assets of different indian processed teas: a comparative approach $\begin{array}{lllll}\text { American Journal of Plant Sciences, } & 8(07), & 1607\end{array}$ https://doi.org/10.4236/ajps.2017.87111

Jeyaraj, A., Zhang, X., Hou, Y., Shangguan, M., Gajjeraman, P., Li, Y., \& Wei, C. (2017). Genome-wide identification of conserved and novel microRNAs in one bud and two tender leaves of tea plant (Camellia sinensis) by small RNA sequencing, microarray-based hybridization and genome survey scaffold sequences. BMC plant biology, 17(1), 212. https://doi.org/10.1186/s12870-017 $\underline{1169-1}$

Kamunya, S., Wachira, F., Pathak, R., Korir, R., Sharma, V., Kumar, R.,

Sharma, R. (2010). Genomic mapping and testing for quantitative trait loci in tea (Camellia sinensis (L.) O. Kuntze). Tree genetics \& genomes, 6(6), 915-929. https://doi.org/10.1007/s11295-010-0301-2

Khan, N., \& Mukhtar, H. (2013). Tea and health: studies in humans. Current pharmaceutical 19(34),

6141-6147 https://doi.org/10.2174/1381612811319340008

Koech, R. K., Malebe, P. M., Nyarukowa, C., Mose, R., Kamunya, S. M., \& Apostolides, Z. (2018). Identification of novel QTL for black tea quality traits and drought tolerance in tea plants (Camellia sinensis). Tree genetics \& genomes, 14(1), 9. https://doi.org/10.1007/s11295-017-1219-8

Kottawa-Arachchi, J. D., Gunasekare, M. K., \& Ranatunga, M. A. (2018), Biochemical diversity of global tea [Camellia sinensis (L.) O. Kuntze] germplasm and its exploitation: a review. Genetic Resources and Crop Evolution, $1-15$.

Liu, M., Tian, H.-1., Wu, J.-H., Cang, R.-R., Wang, R.-X., Qi, X.-H., . . Chen, X.-H. (2015). Relationship between gene expression and the accumulation of catechin during spring and autumn in tea plants (Camellia sinensis L.). Horticulture research, 2, 15011. https://doi.org/10.1038/hortres.2015.11 
Ma, J.-Q., Yao, M.-Z., Ma, C.-L., Wang, X.-C., Jin, J.-Q., Wang, X.-M., \& Chen, L. (2014). Construction of a SSR-based genetic map and identification of QTLs for catechins content in tea plant (Camellia sinensis). PLoS One, 9(3), e93131. https://doi.org/10.1371/journal.pone.0093131

Mondal, T. K., \& Ganie, S. A. (2014). Identification and characterization of salt responsive miRNA-SSR markers in rice (Oryza sativa). Gene, 535(2), 204-209. https://doi.org/10.1016/i.gene.2013.11.033

Ping, S., Zhen-lu, Z., ZHUQiu-fang, Z. G.-y., Ping, X., Yu-ling, L., Zhongxiong, L., \& Jin-ke, L. (2017). Identification of miRNAs and target genes regulating catechin biosynthesis in tea (Camellia sinensis).

Prabu, G., \& Mandal, A. (2010). Computational identification of miRNAs and their target genes from expressed sequence tags of tea (Camellia sinensis). Genomics, proteomics \& bioinformatics, 8(2), 113-121. https://doi.org/10.1016/S1672-0229(10)60012-5

Saravanan, M., John, K. M., Kumar, R. R., Pius, P., \& Sasikumar, R. (2005) Genetic diversity of UPASI tea clones (Camellia sinensis (L.) O. Kuntze) on the basis of total catechins and their fractions. Phytochemistry, 66(5), 561-565. https://doi.org/10.1016/j.phytochem.2004.06.024

Tan, L.-Q., Peng, M., Xu, L.-Y., Wang, L.-Y., Wei, K., Zou, Y., . . . Cheng, H. (2018). The validation of two major QTLs related to the timing of spring bud flush in Camellia sinensis. Euphytica, 214(1), 17. https://doi.org/10.1007/s10681017-2099-6

Tan, L.-Q., Wang, L.-Y., Xu, L.-Y., Wu, L.-Y., Peng, M., Zhang, C.-C., . . Cheng, H. (2016). SSR-based genetic mapping and QTL analysis for timing of spring bud flush, young shoot color, and mature leaf size in tea plant (Camellia sinensis). Tree genetics \& genomes, 12(3), 52. https://doi.org/10.1007/s11295016-1008-9

Tea Advisory Panel, UK. Retrieved from www.teaadvisorypanel.com

Tounekti, T., Joubert, E., Hernández, I., \& Munné-Bosch, S. (2013). Improving the polyphenol content of tea. Critical reviews in plant sciences, 32(3), 192-215. https://doi.org/10.1080/07352689.2012.747384

Varshney, R. K., Graner, A., \& Sorrells, M. E. (2005). Genic microsatellite markers in plants: features and applications. TRENDS in Biotechnology, 23(1), 48-55. https://doi.org/10.1016/j.tibtech.2004.11.005

Zaveri, N. T. (2006). Green tea and its polyphenolic catechins: medicinal uses in cancer and noncancer applications. Life sciences, 78(18), 2073-2080. https://doi.org/10.1016/j.lfs.2005.12.006

Zhao, L.-P., Liu, Z., Chen, L., Yao, M.-Z., \& Wang, X.-C. (2008). Generation and characterization of 24 novel EST derived microsatellites from tea plan (Camellia sinensis) and cross-species amplification in its closely related species and varieties. Conservation Genetics, 9(5), 1327-1331. https://doi.org/10.1007/s10592-007-9476-y 